\title{
Self-imaging with curved gratings
}

\section{Francisco Jose Torcal-Milla *, Luis Miguel Sanchez-Brea, Francisco Javier Salgado-Remacha, Eusebio Bernabeu}

Applied Optics Complutense Group, Optics Department Universidad Complutense de Madrid, Spain Facultad de Ciencias Físicas, Ciudad Universitaria s.n., 28040, Madrid, Spain

\section{A R T I C L E I N F O}

\section{Article history:}

Received 18 March 2010

Received in revised form 31 May 2010

Accepted 1 June 2010

\section{Keywords:}

Diffraction gratings

Talbot effect

Self-imaging

Curved gratings

\begin{abstract}
A B S T R A C T
We analyze the near field behavior of binary amplitude gratings which present a curved profile. This configuration has an important application in rotary optical encoders. This kind of encoders is used to measure the angular displacement between two different parts of the devices. To our knowledge, its behavior in the near field has not been analyzed yet. We have found that Talbot effect is produced but the period of the self-images and Talbot distances change as we separate from the grating. We have fabricated a curved grating and have performed the experiment to corroborate the behavior theoretically found. This fact could be useful in systems that use Talbot effect to measure displacements, since the mechanical tolerances grow.
\end{abstract}

(c) 2010 Elsevier B.V. All rights reserved.

\section{Introduction}

Due to the long range of applications which involve diffraction gratings, they represent an interesting and active field of research since its discovery at the beginning of the 19th century. For example, they can be found in fields of science as different as photonics, chemistry, astrophysics, biology, engineering, and also in diverse applications such as precision optical metrology, telescopes, spectroscopes, machine-tool, etc. [1,2]. Specifically, optical encoders represent an important field of application of diffraction gratings. An encoder is a device able to measure the displacement or relative absolute position between two objects. There are magnetic encoders and optical encoders. Light-diffraction-based optical encoders have been successfully used in machine-tool motion control, since its robustness and precision. These devices are commonly formed by an illumination source, some photo-detectors and two diffraction gratings, whose relative displacement gives the measurement of the movement.

In near field, a Fresnel propagation based model is normally used to describe the behavior of gratings [3]. When a monochromatic plane wave illuminates a diffraction grating, self-images of the grating appear at certain distances after it [3,4]. This effect is known as Talbot self-imaging or Talbot effect and has a crucial role in optical encoders. In a previous work, the authors analyzed the possibility of control of

\footnotetext{
* Corresponding author.

E-mail address: ftorcalmilla@fis.ucm.es (F.J. Torcal-Milla).
}

the position of the Talbot self-images along the propagations axis by controlling the character of the grating, in other words, by controlling its like-phase or like-amplitude behavior [5].

For the ideal case, gratings have been considered as flat or noncurved surfaces, illuminated by a plane and monochromatic wave. This model has been progressively improved introducing new concepts: finite spectral width [6], Gaussian illumination [7], total incoherence [8] or partially coherent light [9-13]. Also non-ideal gratings have been studied: as for example, the effects of surface roughness, [14-17], or surface defects, [18], have been described. These works took into account only flat surfaces.

Effects of curvature over gratings have been also studied [1922 ], but focused to other applications or points of view. Concave or cylindrical gratings have been successfully used in spectrometry, in order to avoid the use of focusing lenses [23,24]. Nevertheless, up to our knowledge there exists no such a study centered on near field diffraction of cylindrical gratings. Cylindrical gratings can be used in annular encoders to measure angular displacements. Our objective is to reach analytical expressions, which lead us to analyze the effect of the grating curvature over the diffraction pattern in near field.

In the present work, we consider a diffraction grating working in reflective mode. The grating is curved over a cylindrical surface, with the strips periodically located along the surface. Assuming that Fresnel approach is valid, the near field propagation of light reflected by cylindrical grating is analyzed and theoretically predicted. Finally, experimental results using cylindrical diffraction gratings engraved by laser ablation are shown, being clear that it is in accordance with theoretical analysis. 


\section{Theoretical approach}

Let us consider the scheme shown in Fig. 1, where a monochromatic plane wave with amplitude $A_{0}$ is propagating from right along the $z$-axis direction and impinges a diffraction grating. The grating presents a cylindrical shape. In Fig. $1,(\xi, z)$ are the coordinates centered at the point in which the grating crosses the $z$-axis, $P$ is a plane perpendicular to the propagation direction placed at the origin of coordinates, $L$ is the projection of the grating length over the $\xi$-axis, $\delta(\theta)$ is the distance between every point of grating and the plane $P$, taking it perpendicularly to the $\mathrm{z}$-axis, and $R$ is the curvature radius of the grating. The radius is defined positive when the center of curvature is placed at the left side of the grating.

We consider an amplitude grating working in reflection configuration, since they are normally engraved over steel tapes [14]. To calculate the diffraction pattern produced by the grating, we use a partially geometrical approach. At the plane $P$ the wave remains as a plane wave, but from this plane to the grating, every point along the $\xi$ axis covers different distances to the curved surface, so it is affected by different optical paths. From Fig. 1 we calculate the optical path for every ray at $\xi$, resulting $\delta(\theta)=R(1-\cos \theta)$. Then the phase change for every point $\xi(\theta)=R \sin \theta$ corresponds to

$\Delta(\theta)=\exp [i k R(1-\cos \theta)]$. results

Performing a variable change to Cartesian coordinates, Eq. (1)

$\Delta(\xi)=\exp \left\{i k R\left[1-\sqrt{1-\left(\frac{\xi}{R}\right)^{2}}\right]\right\}$.

On the other hand, the periodicity of the diffraction grating is no longer linear but angular. Then, its reflectance can be expressed as a Fourier series expansion depending on $\theta$,

$t(\theta)=\sum_{n} a_{n} \exp \left(i q_{\theta} n \theta\right)$

being $a_{n}$ the Fourier coefficients with $n$ integer, $q_{\theta}=2 \pi / p_{\theta}$ and $p_{\theta}$ the angular period whose relation with the linear period is $p_{\theta}=p / R$.

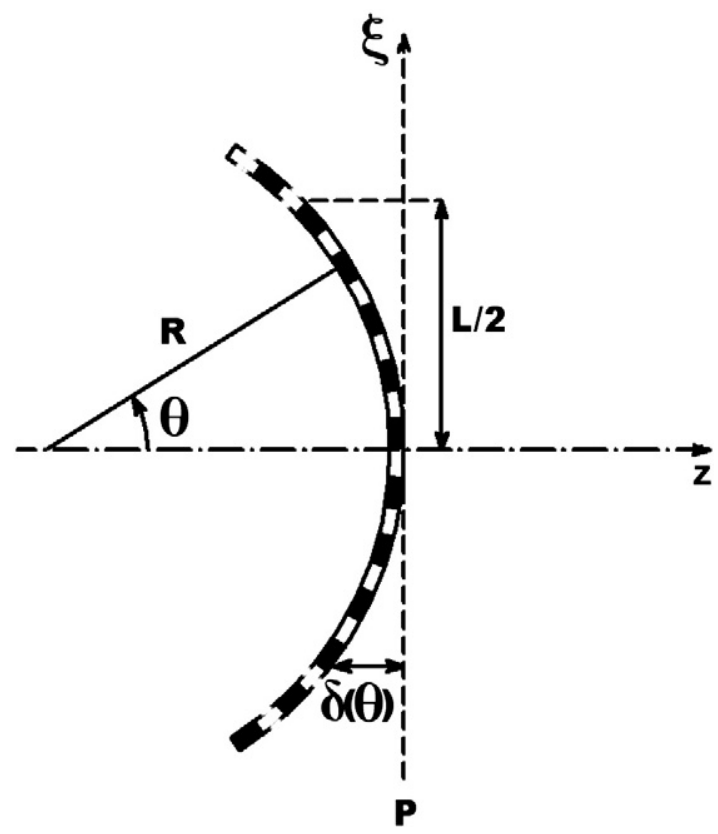

Fig. 1. Curved grating, showing the parameters involved.
Performing the variable change to Cartesian coordinates, Eq. (3) results

$t(\xi)=\sum_{n} a_{n} \exp \left[i q_{\theta} n \arcsin \left(\frac{\xi}{R}\right)\right]$.

Considering Eqs. (2) and (4), the field at the plane $P$, after being diffracted by the grating is

$U_{1}(\xi, z=0)=A_{0} \sum_{n} a_{n} \exp \left\{2 i k R\left[1-\sqrt{1-\left(\frac{\xi}{R}\right)^{2}}\right]\right\} \exp \left[i q_{\theta} n \arcsin \left(\frac{\xi}{R}\right)\right]$,

that is given by the product of the incident field, the reflectance of the grating and the phase difference corresponding to every ray. Since light covers twice the distance from the plane $P$ to the grating due the reflective configuration, $\delta(\theta)$, the optical path doubles its value.

To calculate the field propagation from the plane $P$ forward, we use the Fresnel approach,

$U_{2}(x, z)=\frac{\exp (i k z)}{\sqrt{i k z}} \int_{-L / 2}^{L / 2} U_{1}(\xi, z=0) \exp \left[\frac{i k(x-\xi)^{2}}{2 z}\right] d \xi$,

where $x$ is coordinate perpendicular to the propagation axis at the observation planes, $\lambda$ the illumination wavelength and $k=2 \pi / \lambda$. To solve Eq. (6) we consider that the radius is much larger than the illuminated length of the grating, $R>>L$, and perform Taylor expansions in the exponents of Eq. (5). Taking into account only up to second power in both Taylor expansions, the integral to solve is

$U_{2}(x, z)=A_{0} \frac{\exp (i k z)}{\sqrt{i k z}} \sum_{n} a_{n} \int_{-L / 2}^{L / 2} \exp \left[i k R\left(\frac{\xi}{R}\right)^{2}\right] \exp \left(i q_{\theta} n \frac{\xi}{R}\right) \exp \left[\frac{i k(x-\xi)^{2}}{2 z}\right] d \xi$.

Then the field amplitude at near distances results

$$
\begin{aligned}
U_{2}(x, z)= & \frac{A_{0}}{2} \sqrt{\frac{R}{R+2 z}} \exp \left(i k z+\frac{i k x^{2}}{R+2 z}\right) \sum_{n} a_{n} \exp \left(\frac{i n q_{\theta} x}{R+2 z}\right) \exp \left(-\frac{i n^{2} q_{\theta}^{2} z}{2 k R(R+2 z)}\right) \\
& \times\left(\operatorname{erf}\left\{\frac{(1-i)\left[k R(L+2 x)+2 z\left(k L-n q_{\theta}\right)\right]}{4 \sqrt{k z R(R+2 z)}}\right\}\right. \\
& \left.+\operatorname{erf}\left\{\frac{(1-i)\left[k R(L-2 x)+2 z\left(k L+n q_{\theta}\right)\right]}{4 \sqrt{k z R(R+2 z)}}\right\}\right),
\end{aligned}
$$

where $\operatorname{erf}(\gamma)$ is the error function, defined by $\operatorname{erf}(\gamma)=$ $(2 / \sqrt{\pi}) \int_{0}^{\gamma} \exp \left(-\kappa^{2}\right) d \kappa$.

To understand better the behavior of the grating, we calculate the intensity just performing $I_{2}(x, z)=U_{2}(x, z) U_{2}^{*}(x, z)$. Thus, it results

$$
\begin{aligned}
I_{2}(x, z)= & \frac{I_{0}}{4} \frac{R}{R+2 z} \sum_{n, n^{\prime}} a_{n} a_{n^{\prime}}^{*} \exp \left(\frac{i\left(n-n^{\prime}\right) q_{\theta} x}{R+2 z}\right) \exp \left(-\frac{i\left(n^{2}-n^{\prime 2}\right) q_{\theta}^{2} z}{2 k R(R+2 z)}\right) \\
& \times\left(\operatorname{erf}\left\{\frac{(1-i)\left[k R(L+2 x)+2 z\left(k L-n q_{\theta}\right)\right]}{4 \sqrt{k z R(R+2 z)}}\right\}\right. \\
& \left.+\operatorname{erf}\left\{\frac{(1-i)\left[k R(L-2 x)+2 z\left(k L+n q_{\theta}\right)\right]}{4 \sqrt{k z R(R+2 z)}}\right\}\right) \\
& \times\left(\operatorname{erf}\left\{\frac{(1+i)\left[k R(L+2 x)+2 z\left(k L-n^{\prime} q_{\theta}\right)\right]}{4 \sqrt{k z R(R+2 z)}}\right\}\right. \\
& \left.+\operatorname{erf}\left\{\frac{(1+i)\left[k R(L-2 x)+2 z\left(k L+n^{\prime} q_{\theta}\right)\right]}{4 \sqrt{k z R(R+2 z)}}\right\}\right) .
\end{aligned}
$$

Basically, the effect of curvature is present into the exponential factors producing a variation on the longitudinal and transversal periods of the self-images. The other factors are due to that the grating 
length is finite. In Fig. 2a we show the intensity for a grating with $R=50 \mathrm{~mm}, p=20 \mu \mathrm{m}, L=1 \mathrm{~mm}, \lambda=.6328 \mu \mathrm{m}$ and $n, n^{\prime}=-3,-1,0$, 1,3 . The Fourier coefficients of the grating correspond to the plane grating and are given by $a_{n}=\operatorname{sinc}(n \pi / 2)$. The Talbot self-images $\mathrm{x}$ periodicity varies, increasing in terms of the distance between grating and observation plane. For visual comparison, the near field diffraction pattern produced by a grating with the same characteristics but without curvature $(R \rightarrow \infty)$ is shown in Fig. 2 b.

From the first exponential factor of Eq. (9), the transversal period of the self-images, $\hat{p}$, depends on the radius and the distance from the grating in the following way

$$
\hat{p}=p\left(1+\frac{2 z}{R}\right)
$$

This transversal period increases linearly along distance from the grating for a fixed radius. As it was expected, $\hat{\mathrm{p}}=p$ for $R \rightarrow \infty$.
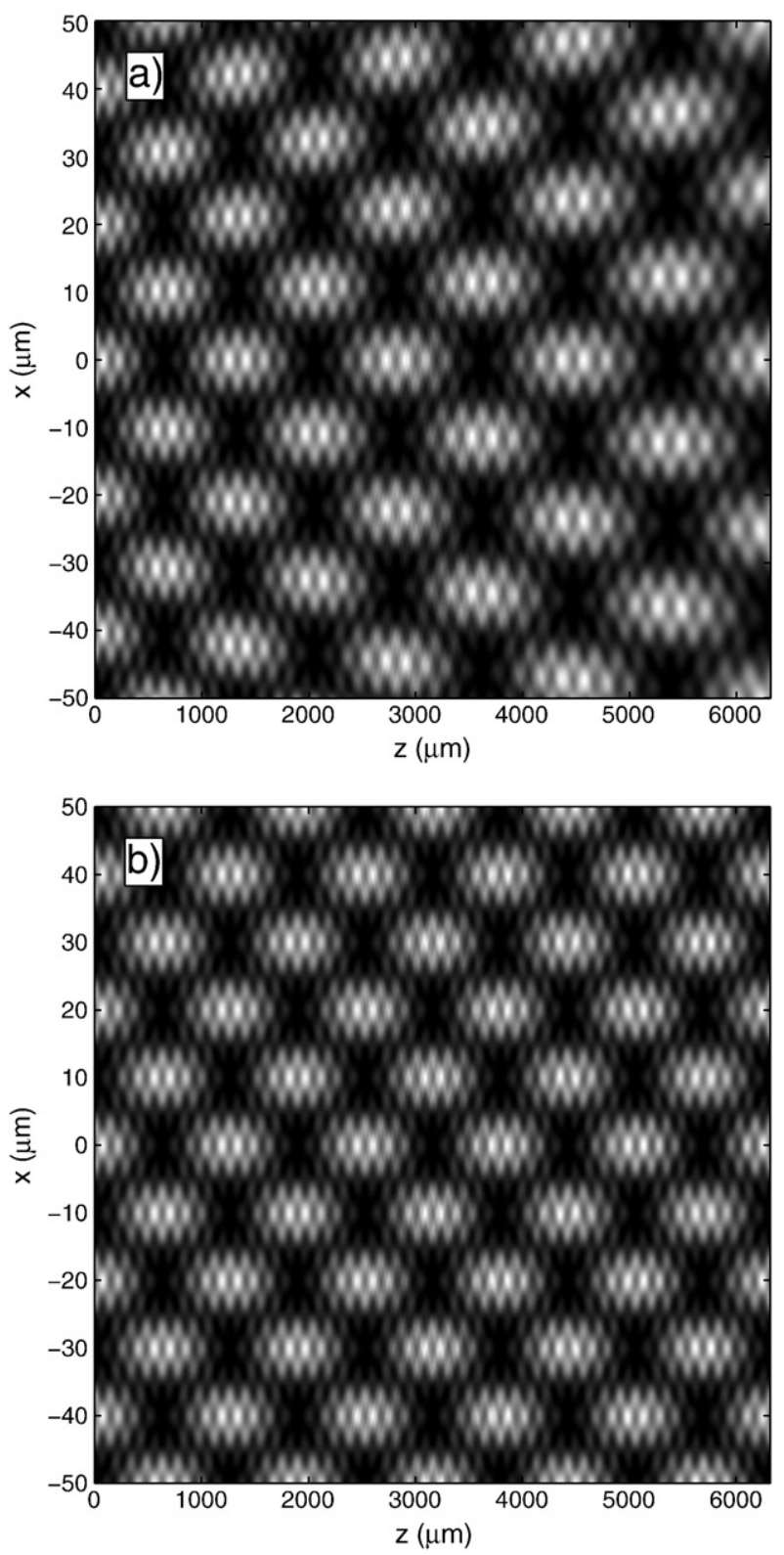

Fig. 2. Analytical self-images for a grating with period $p=20 \mu \mathrm{m}$, illuminated projection of the grating $L=1 \mathrm{~mm}$, illumination wavelength $\lambda=0.6328 \mu \mathrm{m}$ and taking diffraction orders $n, n^{\prime}=(-3,-1,0,1,3)$, a) $\mathrm{R}=50 \mathrm{~mm}$ and b) $R \rightarrow \infty$,
In Fig. 3 we show the profiles corresponding to the first seven selfimages of Fig. 2a. To observe more clearly the effect, we have separated between even (Fig. 3a) and odd (Fig. 3b) self-images. As it can be observed, the period of the self-image increases with the order.

In addition, the Talbot distance variation follows a quadratic function

$\hat{z}_{T}=z_{T}\left(1+\frac{2 z}{R}\right)^{2}$

We show in Fig. 4a the profile along the $z$-axis for $x=0$, corresponding with Fig. 2, the dashed line corresponds to a plane grating and the solid line to a curved grating with $R=50 \mathrm{~mm}$. In addition, we show in Fig. $4 \mathrm{~b}$ the position of the self-images in terms of the order of self-image for the same cases of Fig. 4a. We can observe that the position of the self-image separates from a straight line following a quadratic dependence.

a)
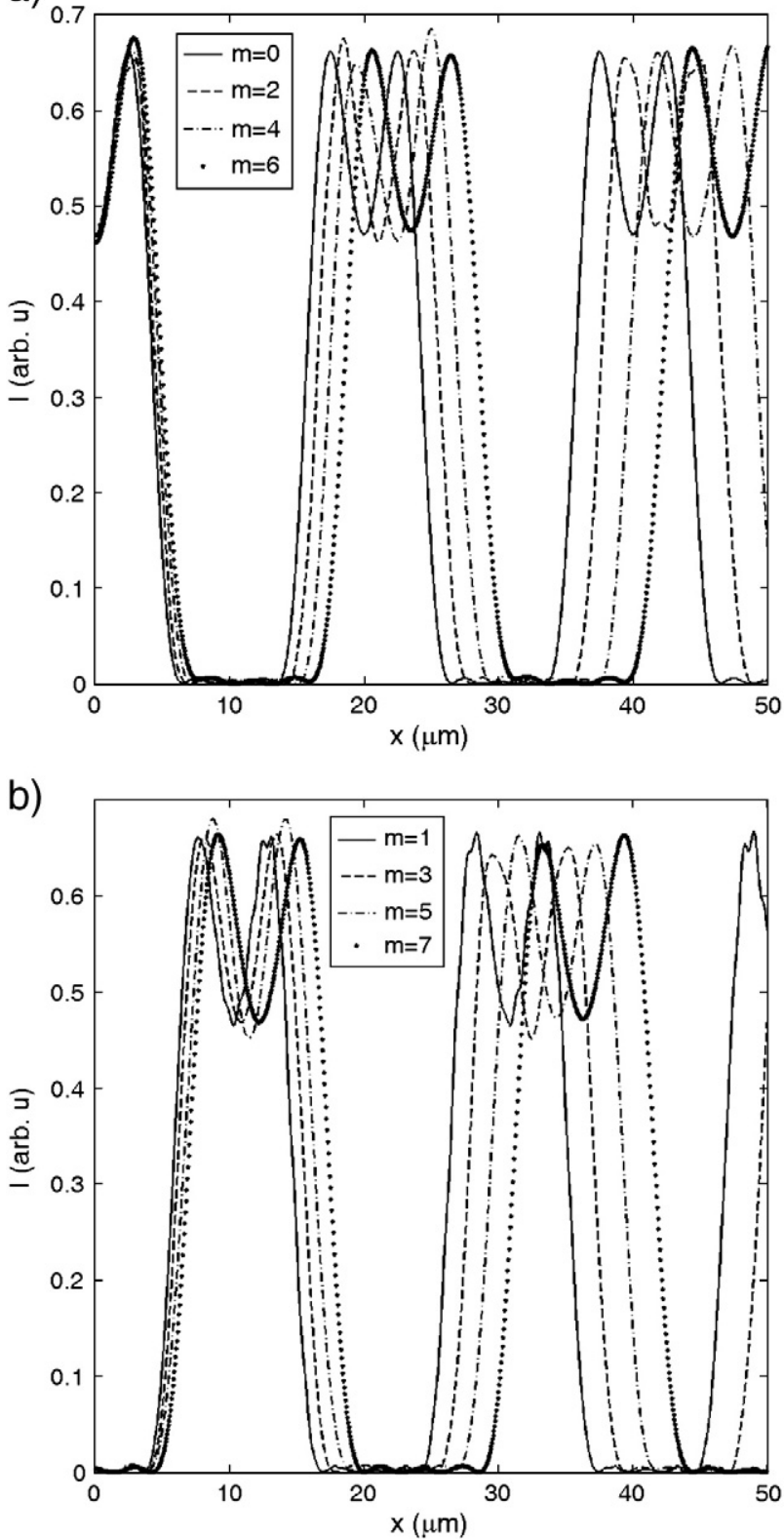

Fig. 3. Profile of the first seven self-images corresponding to Fig. 2a, a) even self-images and $b$ ) odd self-images. 
To finally corroborate the consistence of Eq. (9) with previous results, we carry out the limit for $R \rightarrow \infty$, which corresponds to plane grating, resulting

$$
\begin{aligned}
I_{2}(x, z)= & \frac{I_{0}}{4} \sum_{n, n^{\prime}} a_{n} a_{n^{\prime}}^{*} \exp \left[\frac{i\left(n-n^{\prime}\right) 2 \pi x}{p}\right] \exp \left[-\frac{i\left(n^{2}-n^{\prime 2}\right)(2 \pi)^{2} z}{2 k p^{2}}\right] \\
& \times\left(\operatorname{erf}\left[\frac{(1-i) k(L+2 x)}{4 \sqrt{k z}}\right]+\operatorname{erf}\left[\frac{(1-i) k(L-2 x)}{4 \sqrt{k z}}\right]\right) \\
& \times\left(\operatorname{erf}\left[\frac{(1+i) k(L-2 x)}{4 \sqrt{k z}}\right]+\operatorname{erf}\left[\frac{(1+i) k(L-2 x)}{4 \sqrt{k z}}\right]\right) .
\end{aligned}
$$

a)

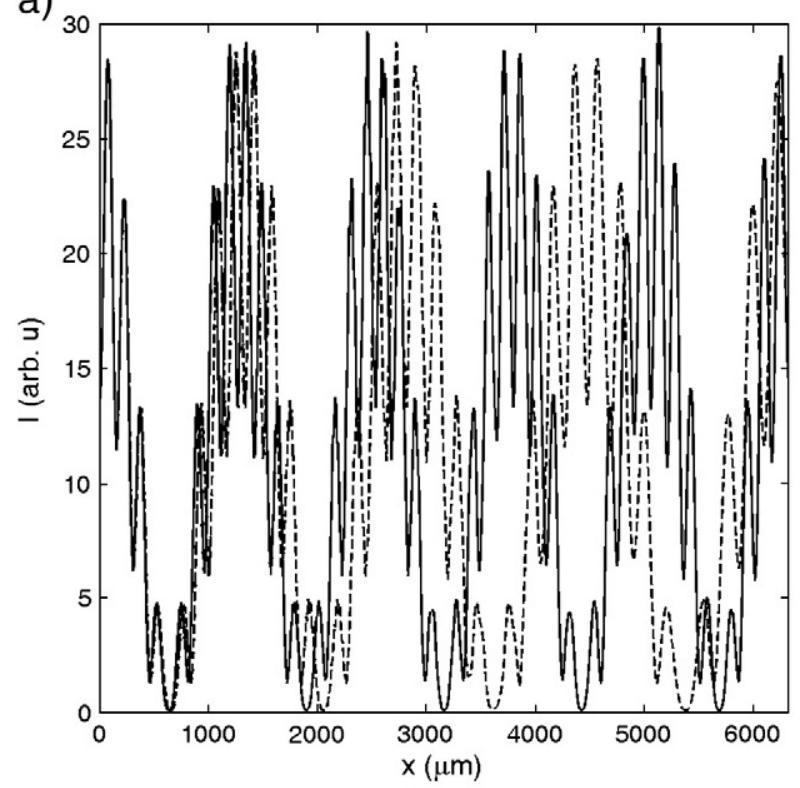

b)

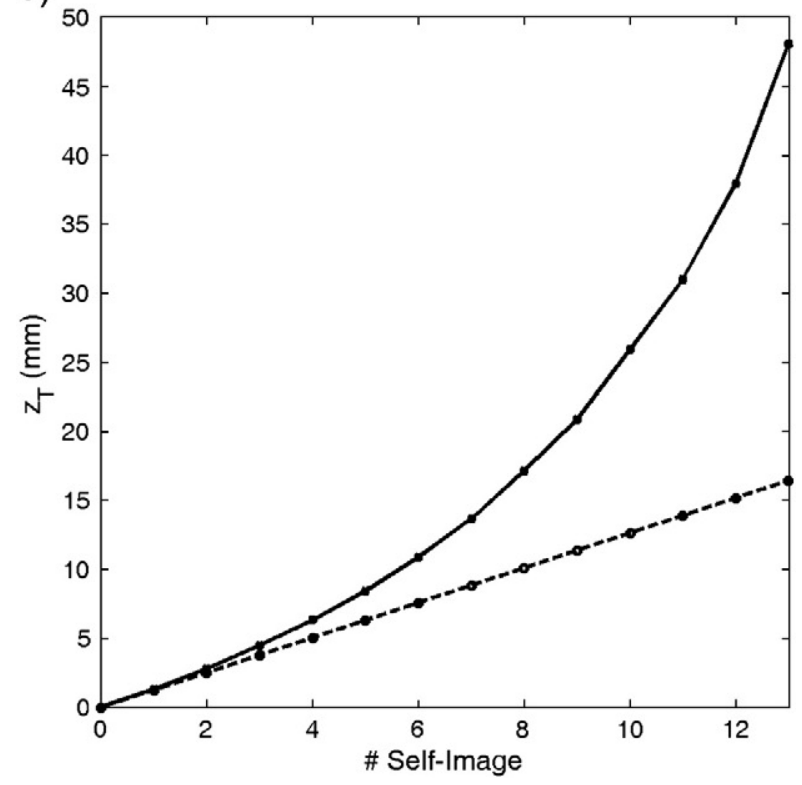

Fig. 4. a) Profile of the self-imaging process (Fig. 2) for $x=0$ along the $z$-axis, plane grating (dashed line), curved grating (solid line), b) behavior of the Talbot distance, $z_{T}$, in terms of the order of self-image, curved grating $(R=50 \mathrm{~mm})$ (solid line), and plane grating (dashed line).

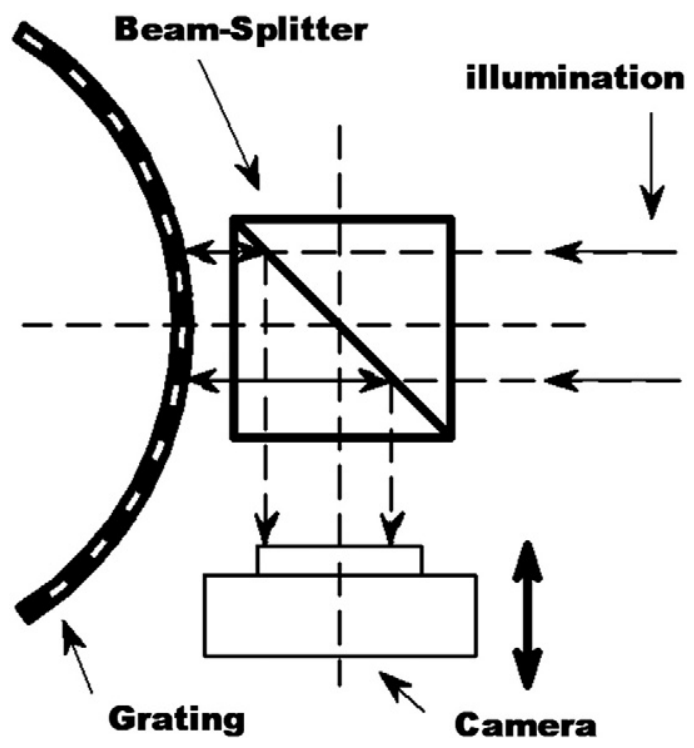

Fig. 5. Scheme of the experimental set-up.

Finally, considering $L \rightarrow \infty$, the classical expression for Talbot effect is recovered, [4],

$I_{2}(x, z)=I_{0} \sum_{n, n^{\prime}} a_{n} a_{n^{\prime}}^{*} \exp \left[\frac{i\left(n-n^{\prime}\right) 2 \pi x}{p}\right] \exp \left[-\frac{i\left(n^{2}-n^{\prime 2}\right)(2 \pi)^{2} z}{2 k p^{2}}\right]$

since $\operatorname{erf}(\gamma \rightarrow \infty) \rightarrow 1$.

\section{Experimental approach}

To corroborate the theoretical analysis carried out in the previous section, we have manufactured a diffraction grating over a flexible steel substrate using a laser ablation system emitting nanosecond pulses with a maximum power peak of $2 \mathrm{~W}$ at $355 \mathrm{~nm}$. The period of the grating is $p=20 \mu \mathrm{m}$. The nature of the substrate allows curving the grating, giving a cylindrical shape. The radius given to the grating is $R=50 \mathrm{~mm}$. The set-up is shown in Fig. 5, where a collimated light beam $(\lambda=.6328 \mu \mathrm{m})$ reaches the curved grating. Since the grating acts in reflection configuration, a beam-splitter becomes necessary. Afterwards, light is captured by a CMOS camera ( $\mu$ eye, pixel size $6 \times 6 \mu \mathrm{m}$ ) coupled to a microscope objective, which is used to magnify the fringes. The camera is free to travel along the propagation axis. The experiment consists of displacing the camera from the grating plane along the propagation axis, acquiring images of the diffracted intensity at different planes. The use of a beam-splitter makes impossible to measure the first Talbot planes but the self-images behavior can be still observed. In Fig. 6 the experimental measurements for a curved (Fig. 6a) and a plane (Fig. 6b) grating are shown. We have included into the images two straight lines to observe the increasing of the period. As can be observed in (Fig. 6a), the curvature of the grating produces changes into the period of the self-images and the Talbot distance, as it was found theoretically. On the other hand, in Fig. $6 \mathrm{~b}$ both lines are parallel, which reveals that the period of the fringes does not change for a plane grating. Due to experimental adjustments, the self-images are not exactly parallel to the $\mathrm{x}$-axis, but the results corroborate the theoretical analysis.

\section{Conclusions}

In this work we have analyzed the near field diffraction pattern of curved diffraction gratings. We have theoretically obtained and 

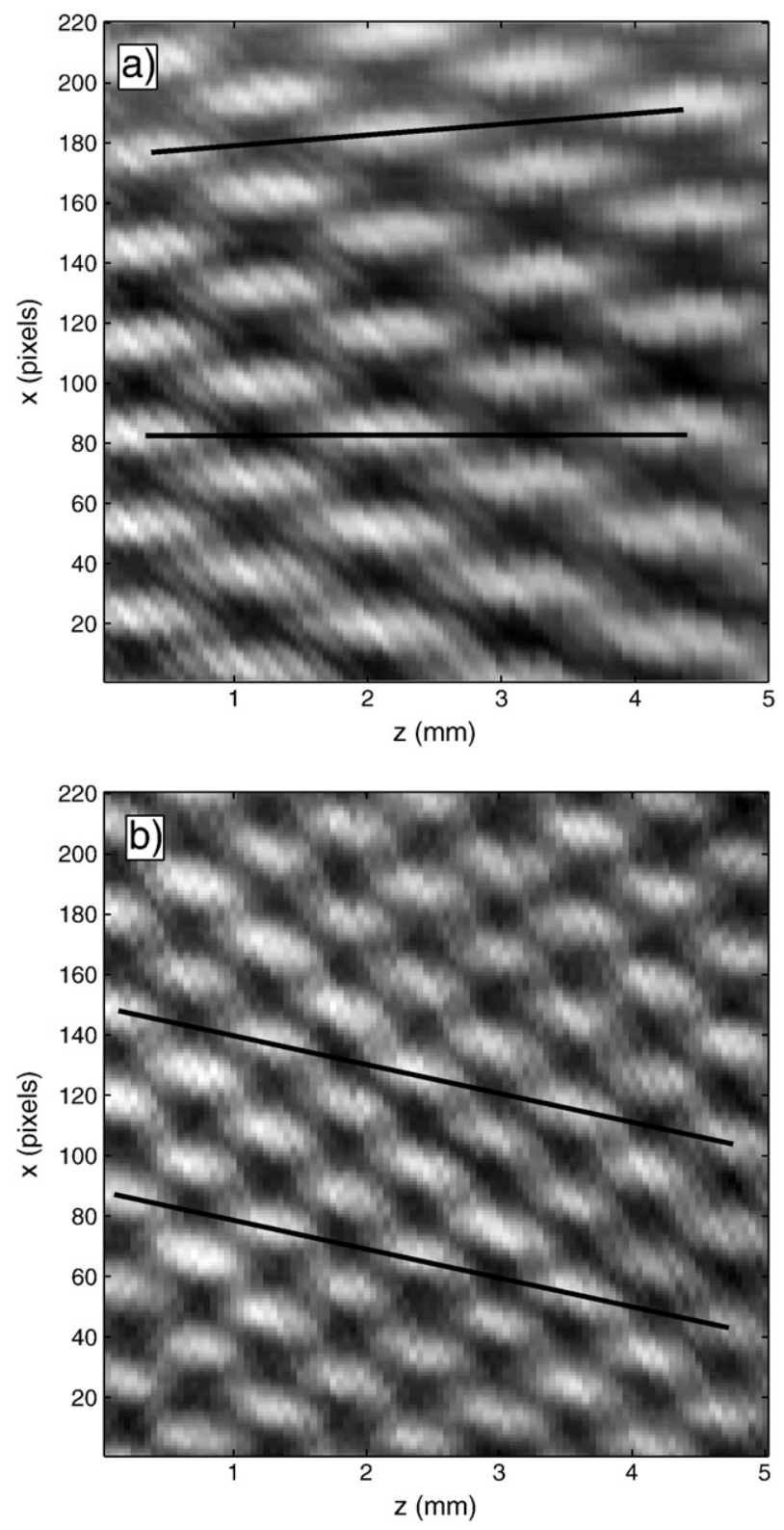

Fig. 6. Experimental intensity distribution after the gratings, $p=20 \mu \mathrm{m}, \lambda=0.6328 \mu \mathrm{m}$, a) $R=50 \mathrm{~mm}$ and b) $R \rightarrow \infty$.

experimentally corroborated that the self-images change their period in terms of the separation from the grating, and also change the Talbot distance between self-images increasing.
To verify the theoretical results, we have fabricated a grating and measured experimentally the self-images when the grating is curved, comparing the result with the self-images formed when the grating is plane. The good agreement between theoretical predictions and experimental results shows that the analytical formalism obtained is correct.

\section{Acknowledgements}

This work has been supported by project CCG08-UCM/DPI-3952 of Dirección General de Universidades e Investigación de la Consejería de Educación de la Comunidad de Madrid and project DPI2008-02391 of the Ministerio de Ciencia e Innovacion of Spain.

\section{References}

[1] E.G. Loewen, E. Popov, Diffraction Gratings and Applications, Marcel Dekker, New York, 1997.

[2] C. Palmer, Diffraction Grating Handbook, Richardson Grating Laboratory, New York, 2000.

[3] K. Patorski, Prog. Opt. 27 (1989) 1.

[4] W.H.F. Talbot, Philos. Mag. 9 (1836) 401.

[5] F.J. Torcal-Milla, L.M. Sanchez-Brea, F.J. Salgado-Remacha, Appl. Opt. 48 (2009) 6252.

[6] L. Garcia-Rodriguez, J. Alonso, E. Bernabeu, Opt. Express 12 (2004) 2529.

[7] L.M. Sanchez-Brea, F.J. Torcal-Milla, E. Bernabeu, Opt. Commun. 281 (2008) 5647.

[8] G.J. Swanson, E.N. Leith, J. Opt. Soc. Am. A 2 (1985) 789.

[9] A. Olszak, L. Wronkowski, Opt. Eng. 36 (1997) 2149

[10] J. Tu, L. Zhan, J. Opt. Soc. Am. A 9 (1992) 983.

[11] D. Crespo, J. Alonso, E. Bernabeu, Appl. Opt. 41 (2002) 1223.

[12] S. Teng, L. Liu, J. Zu, Z. Luan, D. Liu, J. Opt. Soc. Am. A 20 (9) (2003) 1747.

[13] D. Crespo, J. Alonso, E. Bernabeu, J. Opt. Soc. Am. A 17 (2000) 1231.

[14] F.J. Torcal-Milla, L.M. Sanchez-Brea, E. Bernabeu, Appl. Opt. 46 (2007) 3668

[15] L.M. Sanchez-Brea, F.J. Torcal-Milla, E. Bernabeu, Opt. Commun. 278 (2007) 23.

[16] L.M. Sanchez-Brea, F.J. Torcal-Milla, E. Bernabeu, J. Opt. Soc. Am. A 25 (4) (2008) 828.

[17] F.J. Torcal-Milla, L.M. Sanchez-Brea, E. Bernabeu, J. Opt. Soc. Am. A 25 (10) (2008) 2390.

[18] L.M. Sanchez-Brea, F.J. Torcal-Milla, Appl. Opt. 49 (2010) 2190.

[19] H.G. Beutler, J. Opt. Soc. Am. 35 (5) (1945) 311.

[20] H. Haber, J. Opt. Soc. Am. 40 (3) (1950) 153.

[21] C.T. Hsieh, C.K. Lee, App. Opt. 38 (22) (1999) 4743.

[22] X. Prieto-Blanco, C. Montero-Orille, H. Gonzalez-Núñez, M.D. Mouriz, E. López Lago, R. de la Fuente, J. Opt. Soc. Am. A 26 (11) (2009) 2400.

[23] M. Born, E. Wolf, Principles of Optics, Pergamon Press, Oxford, 1980.

[24] B.E. Woodgate, J. Opt. Soc. Am. 64 (5) (1974) 654. 